\title{
Use of clomiphene to prevent premature luteinizing hormone surge during controlled ovarian hyper stimulation
}

\section{Shilpa Bhandari*, Ishita Ganguly, Priya Bhave Chittawar, Pallavi Agarwal, Aparna Singh, Nitika Gupta}

Department of Reproductive Medicine and Surgery, Sri Aurobindo Medical College and PG Institute, Indore, MP, India

Received: 09 April 2016

Revised: 07 May 2016

Accepted: 10 May 2016

\section{*Correspondence: \\ Dr. Shilpa Bhandari, \\ E-mail: drshilpa.b@gmail.com}

Copyright: () the author(s), publisher and licensee Medip Academy. This is an open-access article distributed under the terms of the Creative Commons Attribution Non-Commercial License, which permits unrestricted non-commercial use, distribution, and reproduction in any medium, provided the original work is properly cited.

\section{ABSTRACT}

Background: This study seeks to evaluate concurrent use of clomiphene to block spontaneous LH surge in controlled ovarian stimulation in patients with good ovarian reserve.

Methods: Thirty five fertile donors with good antral count were stimulated from day 1 or 2 of spontaneous or withdrawal bleeds with clomiphene citrate and recombinant FSH. Transvaginal oocyte retrieval was done after 34 hours of trigger. An LH surge was defined as an $\mathrm{LH}$ level $\geq 10 \mathrm{mIU} / \mathrm{ml}$ with progesterone elevation $(\geq 1.0 \mathrm{ng} / \mathrm{ml})$. Fertilization and Embryo quality was assessed from day 2 onwards. Each recipient was transferred a maximum of 3 embryos and remaining were cryopreserved.

Results: After an average stimulation of 8.65 days, 15.4 oocytes were retrieved per stimulation. LH surge was noted in 3 cases $(9.68 \%)$ though oocyte retrieval was done in all cases. Fresh embryo transfer was done in respective recipients of all cases with an implantation rate of $61.29 \%$ and a cumulative live birth rate of $42 \%$.

Conclusions: Controlled ovarian stimulation using clomiphene and gonadotropin is a viable option to prevent LH surge without additional use of antagonist.

Keywords: Clomiphene citrate, LH surge, In vitro fertilization, Ovarian stimulation

\section{INTRODUCTION}

Louise Brown, the first baby born from In Vitro Fertilization (IVF) in 1978 was conceived by oocyte retrieval in a natural cycle. However, natural cycle IVF was soon replaced by controlled ovarian hyper stimulation. For 35 years now, ovarian stimulation has been applied with the aim of increasing the number of oocytes in order to compensate for inefficiencies of the IVF procedure enabling the selection of one or more embryos for transfer. The primary aim of ovarian stimulation is multi-follicular development while inhibiting ovulation at the same time. While the use of gonadotropins has shown to increase the oocyte yield considerably, the ideal method to prevent premature luteinizing hormone (LH) surge continues to be debated. ${ }^{7}$ Use of Gonadotropin releasing hormone $(\mathrm{GnRH})$ agonist for down regulation has been the mainstream procedure till present day. Use of antagonist for preventing premature surge (antagonist protocol) is now being advocated especially as a part of natural cycle or mild stimulation. ${ }^{2,10}$

The ideal IVF protocol secures a high chance of embryo transfer with a low cancellation rate, a high pregnancy rate, a low intervention level, low risk, few side effects, low costs and practical convenience both for the patient and the clinician.

Natural cycle meets some of these criteria, however this 
was on the expense of a relatively high cancellation rate $(25-75 \%)$ and a low clinical pregnancy rate per started cycle (range 0-23\%), but higher per embryo transfer (range $0-30 \%) .^{5-7}$ The main problems with natural cycle IVF are spontaneous LH surge and ovulation leading to high cancellation rates.

Clomiphene has been used extensively as a part of numerous stimulation protocols variably called soft stimulation, mild stimulation etc. ${ }^{1,2,4,10,11}$ The effects of clomiphene citrate on the reproductive tract especially on endometrium have been a matter of concern for many years. Use of gonadotropin with clomiphene, however has been shown to aid in the endometrial development.

Continuation of clomiphene citrate beyond the usual 5 days was also able to inhibit the LH surge. ${ }^{12}$ Thus, a longer stimulation was achieved with clomiphene citrate and premature LH surge was prevented by the antiestrogen effect of clomiphene on the pituitary when the estradiol began to rise. In this pilot study we attempt to explore the feasibility of using clomiphene to block spontaneous LH surge in a controlled ovarian hyperstimulation $(\mathrm{COH})$ without compromising on the oocyte retrieval rate in patients with good ovarian reserve.

\section{METHODS}

This pilot study was conducted in the Department of Reproductive Medicine, Sri Aurobindo Medical College and Post Graduate Institute in the year 2012-2013.During this time period forty two fertile females recruited for the purpose of oocyte donation were selected and counselled regarding the same. The age of donors selected was between 21 to 35 years, with at least 10 antral follicles in both ovaries and 1 spontaneous conception previously. These females were evaluated for their medical fitness to undergo controlled ovarian stimulation. Each female was undergoing $\mathrm{COH}$ for the first time and each donor was stimulated for one specific recipient. Written informed consent was taken regarding the procedure involved, the associated risks, complications. Ethics committee approval was not taken as this was a routine protocol followed in the department, only endocrinological monitoring was done additionally for research purpose \& the cost was bore by the institute.

The oocyte donors were given oral contraceptive pills for a minimum of 12 days from day 3 of their cycles to a maximum of 40 days. In the subsequent cycle, stimulation was started from day 1 or 2 of withdrawal bleed. Baseline evaluation including estradiol (e2), progesterone (p4), and transvaginal sonography (TVS) (for ovarian morphology and endometrial thickness) was done. Stimulation was started with 150 units of recombinant gonadotropin (follicle stimulating hormone $\mathrm{FSH}$ ) if the e2 value was $<50 \mathrm{pg} / \mathrm{ml}$ and endometrial thickness was $<5 \mathrm{~mm}$. clomiphene citrate $50 \mathrm{mg}$ once a day orally was started from first day of stimulation daily till the day of trigger. Follicular study was done on day 5 and dose adjustment was done dependent on follicular recruitment/growth. Ovulation trigger was done using $2 \mathrm{mg}$ leuprolide acetate, when at least 3 follicles were more than $17 \mathrm{~mm}$ diameter. Serum luteinizing hormone (LH), e 2 and $\mathrm{p} 4$ were done on the day of the trigger. An LH surge was defined as an $\mathrm{LH}$ level $\geq 10 \mathrm{mIU} / \mathrm{ml}$ with progesterone elevation $(\geq 1.0 \mathrm{ng} / \mathrm{ml})$. Serum $\mathrm{LH} \geq 10$ $\mathrm{mIU} / \mathrm{ml}$ without an increase in the progesterone level was defined as LH elevation.

Transvaginal oocyte retrieval (TVOR) was done 34 hours after trigger under local anesthesia and sedation in all cases. Intracytoplasmic sperm injection (ICSI) was performed in all cases. Fertilization and embryo quality was assessed from day 2 onwards. Each recipient was transferred a maximum of 3 embryos and remaining were cryopreserved for subsequent thaw transfer. Number of follicle seen, number of oocytes retrieved, number of MII oocytes, embryo fertilized and pregnancy rates were calculated.

\section{RESULTS}

Table 1: Demographic profile of the patients.

\begin{tabular}{|lll|}
\hline & Range & Mean \\
\hline Age & $21-32$ & $26.45 \pm 2.91$ \\
\hline Parity & $1-3$ & $2.26 \pm 0.68$ \\
\hline Antral follicles & $10-24$ & $18.2 \pm 4.13$ \\
\hline Starting E2 & $5-49.7$ & $49.7 \pm 12.26$ \\
\hline Starting P4 & $0.03-0.93$ & $0.45 \pm 0.21$ \\
\hline
\end{tabular}

A total of 42 donors were recruited out of which 35 were fit for stimulation as per protocol. All donors were fertile females with average parity being $2.26 \pm 0.68$. The mean age of donors was 26.4years; range being $21 \mathrm{ys}$ to $32 \mathrm{yrs}$ (Table 1). Transvaginal sonography showed an average of 18 follicles (10 to 24 ) for the entire cohort.

Table 2: Stimulation details.

\begin{tabular}{|l|l|}
\hline \multicolumn{1}{|c|}{ Cycles } & Total/average \\
\hline Oocyte retrieved & 31 \\
\hline \% M2 retrieved & 15.4 \\
\hline Fertilization rate & $76.08 \%$ \\
\hline E2 on day of trigger & 76.1 \\
\hline P4 on day of trigger & 4445 \\
\hline Days of stimulation & 1.37 \\
\hline Total dose & 8.65 \\
\hline
\end{tabular}

The stimulation was started on day 1 or 2 of menstrual period for 35 patients. In 3 patients $(8.5 \%)$ further stimulation was cancelled due to asynchronous or poor follicular growth after first scan on day 5. One patient who did not adhere to the protocol and was not included in further analysis. 
On an average 8.65 days were required for stimulation wherein 15.4 follicles were retrieved per cycle. In 3 out of $31(9.68 \%)$ patients, an LH surge was noted. In all 31 patients oocyte were recovered after TVOR including the ones with LH surge. There was no case of fertilization failure. Embryo transfer was done in all cases in respective recipients.

Table 3: Estradiol levels per follicle and LH surge.

\begin{tabular}{|lllll|}
\hline Number of oocytes & $<10$ & $\mathbf{1 1 - 1 5}$ & $>15$ & P value \\
\hline Cycles & 7 & 12 & 12 & 0.031 \\
\hline E2 per follicle & $424.67 \pm 183.07$ & $323.62 \pm 108.17$ & $237.5 \pm 127.05$ & 0.485 \\
\hline LH surge & 0 & 1 & 2 & 2 \\
\hline
\end{tabular}

Table 4: Estradiol levels and LH surge.

\begin{tabular}{|c|c|c|c|c|}
\hline E2 on day of trigger & $E 2<4000$ & $\mathrm{E} 2>4000$ & Average/total & P value \\
\hline Cycles & 14 & 17 & 31 & \\
\hline LH value & $5.83 \pm 4.69$ & $6.42 \pm 3.37$ & 6.68 & 0.518 \\
\hline LH surge & 1 & 2 & 3 & 0.665 \\
\hline Oocytes retrieved & $14 \pm 5.6$ & $16.53 \pm 5.65$ & 17.75 & 0.186 \\
\hline
\end{tabular}

Table 5: Implantation and pregnancy rate in fresh and frozen thaw cycle.

\begin{tabular}{|llll|}
\hline \multicolumn{1}{|c|}{$\begin{array}{l}\text { Fresh } \\
\text { cycle }\end{array}$} & $\begin{array}{l}\text { Frozen } \\
\text { thaw cycle }\end{array}$ & cumulative \\
\hline Total & 31 & 11 & \\
\hline implantation & $\begin{array}{l}19 \\
(61.29 \%)\end{array}$ & $5(45.45 \%)$ & $24(77.41 \%)$ \\
\hline $\begin{array}{l}\text { Clinical } \\
\text { pregnancy }\end{array}$ & $\begin{array}{l}13 \\
(41.94 \%)\end{array}$ & $5(45.45 \%)$ & $18(58.06 \%)$ \\
\hline Live birth & & & $13(42 \%)$ \\
\hline
\end{tabular}

The average concentration of estradiol on day of trigger and estradiol per follicle was $4445 \mathrm{pg} / \mathrm{ml}$ and 313.13 respectively (Table 2). Severe ovarian hyperstimulation syndrome (OHSS) was not seen in any of the cycles.

Estradiol concentration per follicle decreased as the number of follicles increased. No case of LH surge was seen in patients wherein less than 10 oocytes were retrieved.

In 17 out of $31(54.84 \%)$ patients the estradiol levels were more than $4000 \mathrm{pg} / \mathrm{ml}$ on day of trigger (Table 4). In 1 case where LH surge was seen estradiol levels were less than $4000 \mathrm{pg} / \mathrm{ml}$ whereas in 2 of the cases E2 was more than $4000 \mathrm{pg} / \mathrm{ml}$. Average LH concentration on day of trigger was less in cases with E2 less than $4000 \mathrm{pg} / \mathrm{ml}$.

After oocyte retrieval, $79.9 \%$ oocyte showed MII oocytes. ICSI was performed on all MII oocytes and an approximate $76 \%$ fertilization rate was observed. Approximately 10 embryos (out of which a median of 7 were of grade A) were available for transfer per retrieval cycle.

Fresh embryo transfer was performed in all 31 cycles resulting in implantation in 19 cases $(61.29 \%)$ (Table 5). In 24 patients, a median of 3 (range 0-14) embryos were cryopreserved. Five patients conceived from a total of 11 frozen thaw cycles (implantation rate $45.45 \%$ ).A cumulative of 13 live births was seen.

\section{DISCUSSION}

Soft stimulation protocols were developed to impart a less stressful and less expensive mode of stimulation for patients. One of the important considerations was to reduce the number of injections received by the patient. For this purpose, clomiphene was used in an extended regimen from day 1 of stimulation continuously till the day of trigger. ${ }^{9}$ This caused prolongation of the FSH window and inhibited LH surge simultaneously by competitive estrogen blocking.

Previous studies have used this concept to reduce the amount of gonadotropin used in patients with reduced ovarian reserve. ${ }^{9,11,12}$ In contrast this study was done to evaluate the feasibility of using the same approach in patients with good ovarian reserve and get oocyte yield comparable to standard agonist or antagonist protocol. The mean age of patients in our pilot study is $26.45 \mathrm{yrs}$., which is lower than the usual age of patients seeking IVF treatment. ${ }^{3,4,10}$ However, this pilot study was done for the purpose of developing a simplified stimulation protocol for fertile young oocyte donors. The duplication of 
similar protocol for all infertile patients will have to be validated by larger studies.

Even though this study was not designed to evaluate the convenience of patients, we have found this protocol to be patient friendly as fewer injections were used.

In present cohort LH surge was seen in 3 cases $(9.67 \%)$ which is slightly higher to Teramoto and Kato, wherein ovulation was recorded in $2-3 \%$ with mean $\mathrm{LH}$ values on day of trigger being $7.9( \pm 3.5)$ IU/L. ${ }^{9}$ Williams et al in their retrospective analysis reported a premature $\mathrm{LH}$ surge of $5 \%$ and an oocyte retrieval rate of 3.7 per cycle. ${ }^{11}$ This is comparable to present study even though they did not use clomiphene till the day of trigger. In most of the studies using clomiphene in the initial follicular phase, the main indication of such a protocol was to reduce the gonadotropin requirement. Most of such protocols are hampered by high cancellation rates due to premature LH rise, premature ovulation or reduced chances for successful oocyte retrieval. Therefore most of the natural cycle IVF protocols based on clomiphene; use antagonist cotreatment to reduce the premature LH surge leading to similar oocyte retrieval rates and pregnancy outcomes. $^{10}$

In present study oocytes were retrieved in all cases irrespective of the $\mathrm{LH}$ values on day of trigger. In the study by Teramoto and Kato, the oocyte retrieval rate was $60-70 \%$ in cases where emergency oocyte retrieval was done in view of LH surge. ${ }^{9}$ They also found that the baseline LH values were higher in patients with $\mathrm{LH}$ surge. This difference in finding could be due to small sample size of our study.

In our study $3(8.57 \%)$ cycles were cancelled due to asynchronous growth. In a study by Matsunaga et al, the cycle cancellation rate was $10.6 \%$ with the use of clomiphene, which is comparable to our study. ${ }^{6}$ In most of the soft protocol used, the cancellation rates were in order of $15-30 \%$, which is higher than what we have seen in our study. ${ }^{2,6,7,10}$ This is probably because of small sample size of our study and different patient profile. The patients in our study were those with good ovarian reserve and therefore demonstrated a more synchronous follicular growth than their elder counterparts.

Total estradiol levels on day of trigger are dependent on the number of follicles developed. However estradiol levels per follicle decreased from an average of 313.1 to $237.5(\mathrm{p}=0.03)$ when more than 15 follicles were retrieved, similar to the findings of Teramoto\& Kato 2010. This probably indicates the decrease in oocyte quality with increase in the number of oocytes recruited during stimulation, in turn making the case for softer or milder stimulation stronger.

In cases where the estradiol concentration was lower than $4000 \mathrm{pg} / \mathrm{ml}$, LH levels were more than average. There was no statistical significance in the number of cycles showing LH surge with estradiol concentration. This indicates that higher level of estradiol is also effective in preventing premature rise in LH levels as clomiphene's antagonistic action on the oestrogen receptor strongly inhibits the positive feedback at the level of the hypothalamus. However clomiphene has a half-life of 24 hrs or less and discontinuation of clomiphene as in soft stimulation can cause initiation of LH surge. Present protocol address this issue by continuing clomiphene till the day of trigger and at the same time the presence of endogenous LH contributes to favourable embryonic development. The possibility of using GnRHa for ovulation trigger not only helps promote the cause of OHSS free clinic but also plays an important role in sustaining normal LH dynamics and normal luteal function in the luteal phase of the clomiphene citrate cycle. However it is pertinent to point out that the small sample size of present study precludes us to draw conclusive evidence from these findings and large randomized studies should provide better answers.

In the present series no case of severe OHSS was noted. This could be attributed to agonist trigger as well as the fact that the transfer was done in the recipients $\&$ not the donors who were stimulated.

There continue to be concerns regarding the continued use of clomiphene and its effect on endometrium and ovarian tumor pathogenesis. ${ }^{6}$ In the present study no embryo transfer was done, as the sample population comprised of oocyte donors. Therefore concerns regarding the effect of prolonged use of clomiphene on endometrium remains unaddressed. However transfers in recipients have shown an implantation rate of $(61.29 \%)$ which is comparable to that seen in other studies. ${ }^{8}$ The relatively young age and known fertile potential of the population should be borne in mind when considering the relatively high pregnancy rates in the series.

Median of 3 embryos was available for cryopreservation in 24 patients. 11 frozen thaw cycle were done in 8 patients with an implantation rate of $45.5 \%$, which is slightly lower than that of fresh transfers in the same series. A cumulative live birth rate of $42 \%$ (13/31) was observed in the series. This is comparable to most of the live births reported in the literature by different protocols.

Use of clomiphene has been studied in multiple protocols where it is generally administered from day 2 to day 9 and antagonist is added later to reduce the propensity of LH surge. The current study has demonstrated that it is possible to obtain reasonable results using an IVF protocol with a combination of gonadotropin-Clomiphene citrate for ovarian stimulation without concurrent use of antagonist or agonist. In this study the role of clomiphene is not just to act as anti-estrogen and facilitate increase in endogenous FSH (and thereby reducing the dose requirement) but also to inhibit premature LH surge while maintaining endogenous pituitary function. The concomitant administration of $\mathrm{CC}$ did not influence 
oocyte maturity in terms of metaphase II oocytes. Ideally, the efficiency of the Gn-CC/ICSI protocol should be compared with those obtainable from the current 'gold standard', the long down-regulation protocol or the antagonist protocol in a similar population. Such analyses should be considered in the future, also in terms of costeffectiveness and clients' perceptions.

\section{CONCLUSION}

Preventing premature LH surge remains the aim of ovarian stimulation for ART while procuring maximal good quality of oocytes with minimal gonadotropin dosage. Our pilot study shows the feasibility of use of continuous clomiphene with gonadotropin as an effective tool to prevent premature LH surge without compromising on oocyte yield.

\section{Funding: No funding sources}

Conflict of interest: None declared

Ethical approval: The study was approved by the Institutional Ethics Committee

\section{REFERENCES}

1. Ahmed RY, Mohamed BA, Hesham G, Al- Inany. Clomiphene-acetyl cysteine combination as a new protocol to a friendly IVF cycle. Middle East Fertil Soc J. 2005;10(2):130-4.

2. Frederikke BL, Gitte JA, Svend L. Low Ovarian Stimulation Using Tamoxifen/FSH Compared to Conventional IVF: A Cohort Comparative Study in Conventional IVF Treatments. Reprod Sys Sexual Disorders. 2013;S2:005

3. Gibreel A, Maheshwari A, Bhattacharya S. Clomiphene citrate in combination with gonadotropins for controlled ovarian stimulation in women undergoing in vitro fertilization. Cochrane Database Syst Rev. 2012;11:CD008528.
4. Hojgaard A, Ingerslev HJ, Dinesen J. Friendly IVF: patient opinions. HumReprod. 2001;16(7):1391-6.

5. Li TC, Warren MA, Murphy C, Sargeant S, Cooke ID. A prospective, randomised, cross-over study comparing the effects of clomiphene citrate and cyclofenil on endometrial morphology in the luteal phase of normal, fertile women. $\mathrm{Br} \mathrm{J}$ ObstetGynaecol. 1992;99(12):1008-13.

6. Matsunaga R, Kamihata M, Watanabe S, Kuwahata A,Ochi M. Comparison of embryo developmental competence derived from letrozole and clomiphene citrate for ovarian stimulation in elderly infertile women.FertilSteril. 2013;100(3):S250.

7. Segawa T, Kato K, Miyauchi O, Kawachiya S, Takehara Y, Kato O. Evaluation of minimal stimulation IVF with clomiphene citrate and hMGSegawa. Fertil Steril. 2007;88(1):S286.

8. ShrutiG, Ruma S, Abha M, Shweta M, Neeti T.(2015). Clomiphene based ovarian stimulation in commercial donor program.Journal of Human Reproductive Sciences.8(3):142-145

9. Teramoto S, Kato O. Minimal ovarian stimulation with clomiphene citrate: a large-scale retrospective study. Reproductive BioMedicine Online. 2007;15(2):134-48.

10. Verberg MF, Eijkemans MJ, Macklon NS, Heijnen EM, Baart EB, Hohmann FP. The clinical significance of the retrieval of a low number of oocytes following mild ovarian stimulation for IVF: a meta-analysis. Hum Reprod Update. 2009;15:5-12.

11. Williams SC, Toner JP, Oehninger S, Donahue J, Jones D, Muasher SJ. Minimal Controlled Ovarian Hyperstimulation $(\mathrm{COH})$ Using Clomiphene Citrate/Gonadotropin for IVF/ET: Equivalent Results Compared to the Standard Long Stimulation Protocol with a Significant Reduction in Cost. Fertility and Sterility. 2000;74(3):S230-1.

12. Zhang J, Chang L, Sone Y, Silber S. Minimal ovarian stimulation (mini-IVF) for IVF utilizing vitrification and cryopreserved embryo transfer. Reproductive BioMedicine Online. 2010;21:485-95.

Cite this article as: Bhandari S, Ganguly I, Chittawar PB, Agarwal P, Singh A, Gupta N. Use of clomiphene to prevent premature luteinizing hormone surge during controlled ovarian hyper stimulation. Int J Reprod Contracept Obstet Gynecol 2016;5:1944-8. 